\title{
A pessoa com úlcera de perna, intervenção estruturada dos cuidados de enfermagem: revisão sistemática da literatura*
}

\author{
THE INDIVIDUAL WITH LEG ULCER AND STRUCTURED NURSING CARE \\ INTERVENTION: A SYSTEMATIC LITERATURE REVIEW
}

\section{LA PERSONA CON ÚLCERA DE PIERNA, INTERVENCIÓN ESTRUCTURADA DE LOS CUIDADOS DE ENFERMERÍA: REVISIÓN SISTEMÁTICA DE LA LITERATURA}

\author{
César Fonseca ${ }^{1}$, Tiago Franco², Ana Ramos ${ }^{3}$, Cláudia Silva ${ }^{4}$
}

\section{RESUMO}

Identificar as intervenções de enfermagem à pessoa com úlcera de perna de origem venosa, arterial ou mista. Pesquisa efetuada no motor de busca EBSCO: CINAHL, MEDLINE, com base em artigos em texto integral, publicados entre 2000 e 2010, com os seguintes descritores: Leg* Ulcer* AND Nurs* AND Intervention*, filtrados mediante questão de partida em formato PICO. Simultaneamente, realizada pesquisa na National Guideline Clearinghouse, com a mesma orientação. Uma intervenção centrada na pessoa aumentou os resultados em saúde, variando os cuidados diretos à ferida consoante a etiologia. Como intervenções associadas à cicatrização da úlcera de perna de qualquer etiologia, destacou-se: relação terapêutica enfermeiro/cliente, individualização de cuidados e monitoramento da dor.

\section{DESCRITORES}

Úlcera de perna

Cuidados de enfermagem

Cicatrização

\begin{abstract}
The objective of this study is to identify the nursing interventions for people with venous, arterial or mixed leg ulcers. This study was performed using the EBSCO search engine: CINAHL and MEDLINE yielded results, based on full-text articles published between 2000 and 2010, using the following descriptors: Leg* Ulcer* AND Nurs* AND Intervention*, filtered using a starting question using PICO. At the same time, a search was performed on the $\mathrm{Na}$ tional Guideline Clearinghouse, using the same search guidelines. A person-centered intervention increased positive health outcomes, with a range of direct wound care in agreement with the etiology. The following interventions associated with the healing of leg ulcers of any etiology were highlighted: nurse/client treatment relationship, individualization of care and pain monitoring.
\end{abstract}

\author{
DESCRIPTORS \\ Leg ulcer \\ Nursing care \\ Wound healing
}

\begin{abstract}
RESUMEN
Identificar las intervenciones de enfermería en la persona con úlcera de pierna de origen venoso, arterial o mixto. Se realizó investigación en motores de búsqueda EBSCO, CINAHL, MEDLINE, procurándose artículos en texto integral, publicados entre 2000 y 2010, con los siguientes descriptores: Leg* Ulcer* AND Nurs* AND Intervention*, filtrados mediante pregunta de inicio en formato PICO. Simultáneamente, se realizó investigación en la National Guideline Clearinghouse, con la misma orientación. Una intervención focalizada en la persona aumentó los resultados en salud, variando los cuidados directos a la herida en consonancia con su etiología. Como intervenciones asociadas a la cicatrización de la úlcera de pierna de cualquier etiología, se destacaron: relación terapéutica enfermero/paciente, individualización de cuidados, monitoreo del dolor.
\end{abstract}

\author{
DESCRIPTORES \\ Úlcera en la pierna \\ Atención de enfermería \\ Cicatrización de heridas
}

\footnotetext{
* Artigo escrito originalmente em Português de Portugal. ${ }^{1}$ Doutorando em Enfermagem da Universidade de Lisboa. Investigador Unidade de Investigação \& Desenvolvimento em Enfermagem. Ramada, Portugal. cesar.j.fonseca@gmail.com ${ }^{2}$ Enfermeiro CHLO. Ramada, Portugal. tmoffranco@gmail.com. 3 Enfermeira CHLN. Mestranda em Ciências da Educação. Investigadora Unidade de Investigação \& Desenvolvimento em Enfermagem - Ramada, Portugal. anaramos@esel.pt ${ }^{4}$ Enfermeira CHLN - HPV da Unidade de Cuidados Paliativos da Domus Vida Parque das Nações. Ramada, Portugal. tmoffranco@gmail.com
} 


\section{INTRODUÇÃO}

A enfermagem toma, por foco de atenção, a promoção dos projectos de saúde que cada pessoa vive e persegue. Neste contexto, procura-se ao longo de todo o ciclo vital, prevenir a doença e promover os processos de readaptação após a doença, procura-se a satisfação das necessidades humanas fundamentais e a máxima independência na realização das actividades da vida diária $^{(1)}$. Desta forma, os cuidados de enfermagem ajudam a pessoa a gerir os recursos da comunidade em matéria de saúde, prevendo-se vantajoso o assumir de um papel de pivô no contexto da equipa ${ }^{(1-2)}$.

Paralelamente, o enfermeiro no exercício da sua prática depara-se com desafios cada vez mais exigentes e complexos, como resultado do aumento da esperança média de $\operatorname{vida}^{(3)}$ e, consequente, da prevalência de doenças crónicas, como é o caso da úlcera de perna.

A úlcera de perna pode ser definida como uma ulceração abaixo do joelho em qualquer parte da perna ${ }^{(4)}$, incluindo o pé, sendo classificado como uma ferida crónica, ou seja, uma ferida que permanece estagnada em qualquer uma das fases do processo de cicatrização por um período de 6 semanas ou mais, o que requer uma estruturada intervenção dos cuidados de enfermagem ${ }^{(5)}$. Existem várias etiologias conhecidas da úlcera de perna, sendo as de origem venosa as mais comuns com $70 \%$ dos casos, seguidas as de origem arterial com 10 a $20 \%$ dos casos e as de etiologia mista com 10 a $15 \%$ dos $\operatorname{casos}^{(6)}$. As principais causas do aparecimento de úlceras de perna são a hipertensão venosa crónica, doença arterial ou a combinação das duas anteriores ${ }^{(5-6)}$. As causas menos frequentes são a neuropatia, infecção, vasculites, neoplasias, perturbações sanguíneas e metabólicas, o linfedema e as de origem iatrogénica ${ }^{(5)}$.

A pertinência da presente problemática colhe o seu fundamento no facto de, se estimar que 1,5 a 3 indivíduos em cada 1000 têm uma úlcera de perna, aumentando a prevalência com a idade para 20 em cada 1000 em indivíduos com mais de 80 anos $^{(2,7)}$. A literatura refere que as úlceras de perna são interpretadas como: a forever healing experience $^{(8)}$, em que $40 \%$ dos casos têm uma úlcera de perna durante um ano ou mais, $20 \%$ durante cinco anos ou mais e $45 \%$ têm recidivas, sendo que $35 \%$ dos casos têm quatro ou mais episódios de recidiva(2).

Associado às úlceras de perna existe um elevado consumo de recursos de saúde, tanto em recursos materiais como técnicos, em que aproximadamente 1-2\% do orçamento total de saúde dos países ocidentais ${ }^{(9)}$, dos quais Portugal faz parte, é consumido por clientes com úlcera de perna ${ }^{(4)}$. Adicionalmente, estima-se que $50 \%$ do tempo de trabalho dos enfermeiros da comunidade é investido a prestar cuidados a este tipo de clientes ${ }^{(4,9)}$. Para além do impacto ao nível da economia de saúde, ocorre também uma profunda alteração nas actividades de vida diárias dos indivíduos com este problema ${ }^{(6-8)}$, nomeadamente a presença de dor, limitações na mobilidade, distúrbios do padrão de sono, as alterações na auto-imagem e a incapacidade de desempenhar a actividade laboral, o que gera diminuição dos rendimentos mensais, aumento dos gastos em tratamentos e estimula o isolamento social(10).

\section{MÉTODO}

Como ponto de partida para a revisão sistemática da literatura foi formulada a seguinte questão em formato $\mathrm{PICO}^{(11)}$ :

Em relação à pessoa com úlcera de perna de etiologia venosa, arterial e mista (População), quais as intervenções de enfermagem (Intervenção) que podem influenciar a cicatrização (Outcomes)?

Foi consultado o motor de busca EBSCO, com acesso a duas bases de dados: CINAHL (Plus with Full Text) e MEDLINE (Plus with Full Text), com selecção de artigos em texto integral (04 de Março de 2010), publicados entre $01 / 03 / 2000$ e $01 / 03 / 2010$, com os seguintes descritores: Leg* Ulcer* AND Nurs* AND Intervention*. Obteve-se um total de 114 artigos: 48 artigos na CINAHL e 56 artigos na MEDLINE, com um total final de 7 artigos. Guyatt e Rennie (2002) preconizam que as revisões sistemáticas da literatura devem levar em conta a evidência dos últimos cinco anos. No entanto, considerou-se um período temporal de dez anos, de modo a beneficiar de uma maior abrangência face ao conhecimento existente sobre a matéria em análise ${ }^{(11)}$.

Simultaneamente, foi observada a base de dados electrónica National Guideline Clearinghouse ${ }^{\mathrm{TM}}$ (NGC) onde foram consultadas linhas de orientação para a prática clínica, em modo Detailed Search, (17 de Março de 2010), seleccionando as seguintes opções de pesquisa: Keyword: Leg Ulcer, Intended Users: Nurse, Clinical Specialty: Nursing. Foram obtidas 13 linhas de orientação para a prática clínica, das quais seleccionamos 5 .

Para conhecer os diferentes tipos de produção de conhecimento patentes nos artigos filtrados, utilizaram-se sete níveis de evidência(11): Nível I: Evidência decorrente de Revisões Sistemáticas ou Meta-análise de Estudos Randomizados Controlados (RCT's) relevantes, ou evidência decorrente de Guidelines para a prática clínica, baseadas em revisões sistemáticas de RCT's; Nível II: Evidência obtida através de pelo menos RCT; Nível III: Evidência obtida através de um estudo controlado, sem randomização; Nível IV: Evidência obtida através de estudos de caso-controle ou de corte; Nível V: Evidência obtida através de revisões sistemáticas de estudos qualitativos e descritivos; Nível VI: Evidência obtida através de um único estudo des- 
critivo ou qualitativo; Nível VII: Evidência obtida através da opinião de autores e/ou relatórios de painéis de peri- tos. O processo de pesquisa e selecção do material para análise, explicita-se na Figura 1.

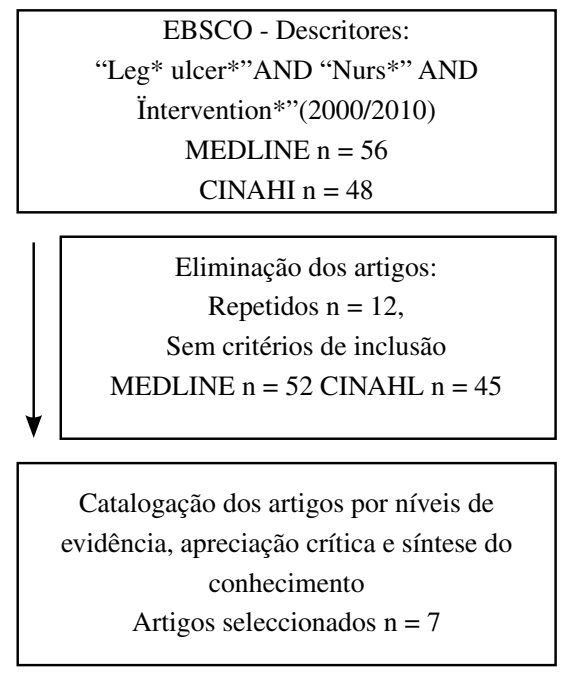

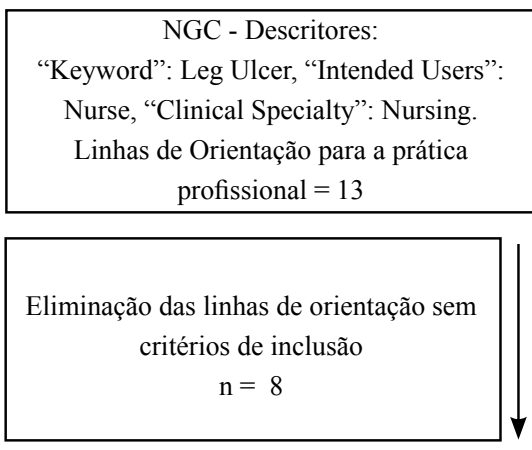

Catalogação das linhas de orientação por níveis de evidência, apreciação crítica e síntese do conhecimento Linhas seleccionadas $n=5$

Corpus de análise

$\mathrm{n}=12$

Figura 1 - Processo de pesquisa e selecção dos artigos - Pesquisa CINAHL, MEDLINE - Período 2000-2010.

\section{RESULTADOS}

Para tornar perceptível e transparente a metodologia utilizada explicita-se a listagem dos 12 artigos selecciona- dos (Quadro 1) para o corpus de análise, que constituíram o substrato para a elaboração da discussão e respectivas conclusões, tendo sido submetidos à classificação por níveis de evidência.

Quadro 1 - Corpus de Análise - Pesquisa CINAHL, MEDLINE - Período 2000-2010

\begin{tabular}{|c|c|c|c|c|}
\hline Nível evidência/ Artigos & Método & Participantes & Intervenções & Resultados \\
\hline Nível de Evidência - V(8) & $\begin{array}{c}\text { Estudo Qualitativo/ } \\
\text { descritivo }\end{array}$ & $\begin{array}{l}50 \text { pessoas idosas com } \\
\text { úlceras de perna de origem } \\
\text { venosa. }\end{array}$ & $\begin{array}{l}\text { Entrevistas } \\
\text { semi-estruturadas }\end{array}$ & $\begin{array}{l}\text { Os idosos com úlceras de perna de origem } \\
\text { venosa esperam sentir-se valorizados } \\
\text { no tratamento e que os seus enfermeiros } \\
\text { cuidadores sejam gentis e profissionais } \\
\text { em quem possam confiar de modo a criar } \\
\text { uma relação de ajuda. }\end{array}$ \\
\hline Nível de Evidência - II(10) & Quantitativo & $\begin{array}{l}56 \text { pessoas com ulcera } \\
\text { de perna de origem venosa }\end{array}$ & $\begin{array}{l}10 \text { enfermeiros fizeram } \\
\text { formação sobre } \\
\text { protocolos } \\
\text { de prevenção e ratamento. }\end{array}$ & $\begin{array}{l}\text { O grupo de intervenção seguindo em } L e g \\
\text { Clubss apresentou melhores resultados } \\
\text { em relação à dor e volução cicatricial da } \\
\text { ulcera que o grupo de controlo. }\end{array}$ \\
\hline Nível de Evidência - V(12) & $\begin{array}{l}\text { Revisão Sistemática } \\
\text { da Literatura }\end{array}$ & $\begin{array}{l}20 \text { artigos relacionados } \\
\text { com pessoas com ulcera de } \\
\text { perna de origem venosa e/ } \\
\text { ou mista. }\end{array}$ & $\begin{array}{l}\text { Foram analisados os } \\
\text { artigos de forma a } \\
\text { conseguir perceber o tipo } \\
\text { de tratamento efectuado e } \\
\text { o que motivou o sucesso } \\
\text { do tratamento. }\end{array}$ & $\begin{array}{l}\text { As pessoas com úlcera de perna de origem } \\
\text { venosa utilizando meias de compressão } \\
\text { de classe III (pressão exercida no maléolo } \\
\text { de de } 25-35 \mathrm{mmHg} \text { ) têm melhores } \\
\text { resultados em comparação com meias } \\
\text { que exercem menos pressão. } \\
\text { A elevação das pernas em doentes que } \\
\text { não utiliza meias ou qualquer outro } \\
\text { sistema de compressão. }\end{array}$ \\
\hline
\end{tabular}

continua... 
continuação...

\begin{tabular}{|c|c|c|c|c|}
\hline Nível evidência/Artigos & Método & Participantes & Intervenções & Resultados \\
\hline Nível de Evidência - V(15) & $\begin{array}{l}\text { Estudo Qualitativo/ } \\
\text { descritivo }\end{array}$ & $\begin{array}{l}25 \text { pessoas com ulcera de } \\
\text { perna de origem venosa } \\
\text { ou mista. }\end{array}$ & $\begin{array}{l}\text { Entrevista semi- } \\
\text { estruturada }\end{array}$ & $\begin{array}{l}\text { A pessoa com úlcera de perna de origem } \\
\text { venosa ou mista necessita de informação } \\
\text { sobre o efeito do exercício físico no } \\
\text { processo de cicatrização das suas } \\
\text { feridas, mas também de ser estimulada a } \\
\text { modificar o seu estilo de vida. }\end{array}$ \\
\hline Nível de Evidência - V(7) & $\begin{array}{l}\text { Revisão Sistemática } \\
\text { da Literatura }\end{array}$ & $\begin{array}{l}31 \text { artigos relacionados } \\
\text { com pessoas com ulcera de } \\
\text { perna de origem venosa e/ } \\
\text { ou mista. }\end{array}$ & $\begin{array}{l}\text { Foram analisados os } \\
\text { artigos de forma a } \\
\text { conseguir perceber a não } \\
\text { adesão ao tratamento. }\end{array}$ & $\begin{array}{l}\text { Dor, desconforto e diferentes estilos } \\
\text { de vida são algumas das razões para } \\
\text { os doentes com úlcera de perna } \\
\text { não aderirem ao tratamento. Os } \\
\text { profissionais de saúde devem focar- } \\
\text { se nos problemas relatados pelos } \\
\text { doentes de modo a conseguir ajudá- } \\
\text { los a ultrapassar esses problemas e a } \\
\text { motivá-los ao tratamento. }\end{array}$ \\
\hline Nível de Evidência - V(4) & $\begin{array}{l}\text { Revisão Sistemática } \\
\text { da Literatura }\end{array}$ & $\begin{array}{l}\text { Publicações sobre o } \\
\text { suporte social e pessoas } \\
\text { com úlceras de perna. }\end{array}$ & $\begin{array}{l}\text { Comparam vários estudos } \\
\text { procurando estabelecer } \\
\text { uma relação entre o efeito } \\
\text { do suporte social na } \\
\text { cicatrização da úlcera de } \\
\text { perna de origem venosa } \\
\text { bem como na recidiva. }\end{array}$ & $\begin{array}{l}\text { O apoio social é muito importante para as } \\
\text { pessoas com ulcera de perna de origem } \\
\text { venosa, sendo esse apoio necessário } \\
\text { quer durante quer após a ferida estar } \\
\text { cicatrizada, de modo a prevenir recidivas. }\end{array}$ \\
\hline Nível de Evidência - II(13) & $\mathrm{RCT}$ & $\begin{array}{l}\text { Todas as pessoas com } \\
\text { úlcera de perna da região } \\
\text { de Skaraborg (Suécia). }\end{array}$ & $\begin{array}{l}\text { Identificação das pessoas } \\
\text { com úlcera de perna, sua } \\
\text { etiologia, prevalência e o } \\
\text { tratamento em curso. }\end{array}$ & $\begin{array}{l}\text { Constatou-se que a úlcera de perna de } \\
\text { origem venosa continua a ser a mais } \\
\text { prevalente seguida da úlcera de origem } \\
\text { arterial. No geral existe uma redução } \\
\text { na prevalência em relação a estudos } \\
\text { anteriores. }\end{array}$ \\
\hline Nível de Evidência - I(16) & $\begin{array}{l}\text { Revisão Sistemática } \\
\text { da Literatura }\end{array}$ & $\begin{array}{l}325 \text { artigos de bases de } \\
\text { dados(Cinahl, Medline e } \\
\text { Cochrane) }\end{array}$ & $\begin{array}{l}\text { Revisão crítica dos artigos } \\
\text { encontrados de modo a } \\
\text { elaborar um conjunto de }\end{array}$ & $\begin{array}{l}\text { São elaboradas recomendações sobre } \\
\text { como avaliar e intervir }\end{array}$ \\
\hline Nível de Evidência - I(16) & & & recomendações & $\begin{array}{l}\text { em doentes com úlcera de perna de } \\
\text { origem arterial, nos seguintes pontos: } \\
\text { desbridamento, escolha do penso, } \\
\text { controlo da infecção, nutrição, controlo } \\
\text { da dor. }\end{array}$ \\
\hline Nível de Evidência - I(16) & $\begin{array}{l}\text { Revisão Sistemática } \\
\text { da Literatura }\end{array}$ & $\begin{array}{l}180 \text { artigos de bases } \\
\text { de dados (Medline e } \\
\text { Cochrane) }\end{array}$ & $\begin{array}{l}\text { Revisão crítica dos artigos } \\
\text { encontrados de modo a } \\
\text { elaborar um conjunto de } \\
\text { recomendações }\end{array}$ & $\begin{array}{l}\text { São elaboradas recomendações sobre } \\
\text { como avaliar, prevenir e tratar de pessoas } \\
\text { com úlcera de perna de origem venosa. }\end{array}$ \\
\hline Nível de Evidência - I(20) & $\begin{array}{l}\text { Revisão Sistemática } \\
\text { da Literatura }\end{array}$ & $\begin{array}{l}210 \text { artigos de bases de } \\
\text { dados (Medline e Embase) }\end{array}$ & $\begin{array}{l}\text { Revisão crítica dos artigos } \\
\text { encontrados de modo a } \\
\text { elaborar um conjunto de } \\
\text { recomendações }\end{array}$ & $\begin{array}{l}\text { São elaboradas recomendações major } \\
\text { sobre como diagnosticar, tratar e gerir a } \\
\text { úlcera de perna de origem venosa. }\end{array}$ \\
\hline Nível de Evidência - I(19) & $\begin{array}{l}\text { Revisão Sistemática } \\
\text { da Literatura }\end{array}$ & $\begin{array}{l}8 \text { guidelines e } 54 \text { estudos } \\
\text { (Cinahl e Medline). }\end{array}$ & $\begin{array}{l}\text { Revisão crítica dos artigos } \\
\text { encontrados de modo a } \\
\text { elaborar um conjunto de } \\
\text { recomendações }\end{array}$ & $\begin{array}{l}\text { São elaboradas } 39 \text { recomendações } \\
\text { sobre avaliação do doente, avaliação e } \\
\text { tratamento da úlcera, gestão da dor e } \\
\text { infecção, aplicação de terapia compressiva } \\
\text { e de outras terapias complementares, } \\
\text { formação para os doentes e organizações } \\
\text { que cuidam essas pessoas. }\end{array}$ \\
\hline Nível de Evidência - V(17) & $\begin{array}{l}\text { Revisão Sistemática } \\
\text { da Literatura }\end{array}$ & $\begin{array}{l}\text { Revisão de } 3 \text { guidelines } \\
\text { (Medline e Cochrane) }\end{array}$ & $\begin{array}{l}\text { Revisão crítica das } \\
\text { guidelines de modo a } \\
\text { elaborar um conjunto de } \\
\text { recomendações }\end{array}$ & $\begin{array}{l}\text { São elaboradas recomendações sobre } \\
\text { como avaliar úlceras de perna, bem como } \\
\text { tratar as diferentes etilogias: venosa, } \\
\text { arterial e mista. }\end{array}$ \\
\hline
\end{tabular}




\section{DISCUSSÃO}

O sucesso no tratamento de pessoas com úlcera de perna está associado com a motivação(2-4,7). Por outro lado, a não adesão ao tratamento de muitos clientes com úlcera de perna de origem venosa e mista está relacionada com presença de dor, o desconforto, a desmotivação, o isolamento social, insuficiente apoio social e a ausência de um estilo de vida saudável, que é repetidamente enfatizado pelos profissionais de saúde, nomeadamente pelo enfermeiro ${ }^{(4,6)}$. Concomitantemente, recomenda-se que o enfermeiro adquira e utilize informação sobre o ambiente/ contexto sociocultural em que o cliente vive, o suporte social existente e a sua qualidade, a profissão que desempenha e os seus hábitos de vida(12-13), bem como uma abordagem centrada no controlo da dor ${ }^{(10)}$, principalmente quando o plano terapêutico envolve compressão no leito da ferida ${ }^{(12-13)}$. Como factor positivamente associado a uma participação activa no plano de cuidados destaca-se a inclusão de pessoas significativas ${ }^{(4)}$, bem como o contacto com pessoas em situação semelhante, ou seja, com pessoas com úlcera de perna tanto em fase activa ou remissiva ${ }^{(2,14-15)}$.

Na Austrália foi introduzido um novo conceito de prestação de cuidados a pessoas com úlcera de perna que assenta na criação de espaços (Leg Clubs)(10), onde enfermeiros com formação específica na área da úlcera de perna, promovem a interacção social entre pessoas com o mesmo tipo de úlcera, avaliam o suporte necessário a cada indivíduo, fazem formação desses clientes no sentido do auto-cuidado e gestão de caso, efectuam o respectivo tratamento e acompanhamento contínuo ${ }^{(4-9)}$. O resultado da implementação deste projecto foi a diminuição da dor, o progresso significativo da cicatrização e o aumento da qualidade de vida, nomeadamente no trabalho, no humor, na mobilidade, no padrão de sono, entre outros aspectos ${ }^{(10)}$. O efeito positivo deste modelo reflecte-se também ao nível social ${ }^{(9)}$, visto que um contacto social mais alargado e com pessoas que têm ou tiveram o mesmo problema, diminui o isolamento social e proporciona mecanismos de coping eficazes para lidar com a situação de crise - a doença ${ }^{(4,16)}$.

Como membro activo de uma equipa multidisciplinar, o enfermeiro não deve actuar isoladamente, devendo criar em conjunto, objectivos e estratégias que promovam uma actuação dirigida às necessidades reais de um determinado indivíduo ${ }^{(16-17)}$. Para tal é necessário que, os enfermeiros se mantenham actualizados, devendo realizar formações na área e manter uma boa comunicação com os seus pares, pois cuidar deste tipo de doenças revela-se mais efectivo se for efectuado em equipa multidisciplinar ${ }^{(12)}$.

A forma como aborda e a atitude que manifesta na prestação de cuidados é algo bastante observado pelos clientes, na medida em que a mobilização de competências de relação interpessoal conduz ao estabelecimento da confiança(17-19), que conjuntamente com uma boa performance técnico-científica, por parte do enfermeiro, gera um processo favorável no processo de cicatrização da ferida(10). O enfermeiro deve então agir de forma a promover o bem-estar, através do estabelecimento de uma relação empática e uma abordagem holística do cliente ${ }^{(20)}$, devendo essa relação manter-se mesmo após a úlcera cicatriza$\mathrm{da}^{(21)}$, dado que alguns clientes referiram não querer a sua úlcera cicatrizada, como forma de manter contacto com o seu enfermeiro prestador de cuidados(4). No estabelecimento da relação interpessoal, o consentimento informado aumentou a confiança nos cuidados de saúde $\mathrm{i}^{(14,17)}$, pois a partir da literacia para a saúde os clientes puderam fazer escolhas livres e responsáveis, com vista à sua autonomia, que favoreceu o empoderamento das pessoas no seu processo de saúde/ doença ${ }^{(10)}$. A comunicação é um instrumento de enfermagem com evidente relevância ${ }^{(15)}$, onde a criação de programas de formação que insiram a área cognitiva, comportamental e afectiva, conjuntamente, trouxeram efectiva vantagem para o cliente ${ }^{(2,7)}$.

Relativamente às linhas orientadoras recomendados, para cuidar uma pessoa com úlcera de perna de origem venosa, arterial ou mista é importante o enfermeiro: conhecer a história clínica da pessoa (antecedentes pessoais, patologias crónicas, estado actual do cliente) e a história da úlcera (origem, tempo, tratamentos efectuados) ${ }^{(6,12,14,20)}$. Ao avaliar minuciosamente as características da ferida (tamanho, profundidade, exsudado, leito da ferida, tipo de tecidos, pele peri-lesional, dor $)^{(14,18)}$, o enfermeiro pode decidir o tratamento sempre em conjunto com o cliente (Quadro 2), de modo a estabelecerem metas comuns $^{(15,18,21-22)}$.

Deste modo, o tratamento deve incidir na prevenção da dor ${ }^{(10)}$, preparação do leito da ferida ${ }^{(6-9)}$, limpeza da ferida(14-15,16), gestão dos produtos a aplicar no leito e pele peri-lesional(7), escolha conjunta do tipo de material para aplicação de terapia compressiva e elaboração de um plano de exercício físico ${ }^{(8-14-18)}$, formação contínua do cliente ${ }^{(23)}$, e referenciação para especialidades em caso de reacções alérgicas ${ }^{(13)}$, necessidade de terapias complementares e/ou tratamentos efectuados não eficazes em que a úlcera/ estado do cliente se deteriora. 
Quadro 2 - Intervenções de enfermagem aos clientes com úlcera de perna de etiologia venosa, arterial e mista- Pesquisa CINAHL, MEDLINE - Período 2000-2010

\begin{tabular}{|c|c|}
\hline ULC & E ETIOLOGIA ARTERIAL \\
\hline $\begin{array}{l}\text { • Aplicar terapia compressiva se Índice de Pres- } \\
\text { são Tornozelo-braço (IPTB) superior a 0,8; } \\
\text { - Escolher o tipo de compressão a utilizar: sis- } \\
\text { tema de compressão multicamada abaixo do jo- } \\
\text { elho (tratamento geral); sistema de compressão } \\
\text { reduzida (em caso de doente não tolerar com- } \\
\text { pressões mais elevadas); meias de compressão } \\
\text { (após ulcera cicatrizada); sistema de compres- } \\
\text { são intermitente (pode ser utilizado isoladamen- } \\
\text { te ou em conjunto com outro sistema de com- } \\
\text { pressão, de forma a aumentar o retorno venoso); } \\
\text { • Escolher o material de terapia compressiva a } \\
\text { utilizar: ligaduras elásticas (longa tracção), con- } \\
\text { sideradas mais eficazes; ligaduras não elásticas } \\
\text { (curta tracção), provocam menos desconforto/ } \\
\text { dor; sistemas de ligaduras elásticas multi-cama- } \\
\text { das (2, } 3 \text { ou } 4 \text { camadas); meias elásticas; meias } \\
\text { elásticas multi-camada; ligadura empragnada } \\
\text { em zinco (Bota de Unna) juntamente com liga- } \\
\text { duras elásticas; } \\
\text { • Aplicar meias de compressão com medida per- } \\
\text { sonalizada, se a úlcera cicatrizou; } \\
\text { • Referenciar para cirurgia vascular nas seguin- } \\
\text { tes situações: ausência de redução das } \\
\text { dimensões a ulcera após } 30 \text { dias de tratamento; } \\
\text { úlcera com mais de } 6 \text { meses; intolerância à tera- } \\
\text { pia compressiva; ineficácia no controlo da dor; } \\
\text { recidivas frequentes. }\end{array}$ & $\begin{array}{l}\text { - Realizar formação para os doentes de forma contínua, abordando os seguintes temas: controlo } \\
\text { das patologias de base; cessação tabágica e etanólica; adesão ao regime terapêutico; estimular a } \\
\text { ingestão de alimentos ricos em vitamina B6 (aumenta o HDL-C e diminui triglicéridos), como por } \\
\text { exemplo: batata, banana, peito de frango, semente de girassol, salmão, atum, abacate, entre outros; } \\
\text { prevenção de traumatismos químicos, térmicos ou mecânicos nos membros inferiores; cuidados à } \\
\text { pele; utilização de calçado adequado e meias não compressíveis; } \\
\text { - Instituir um programa de exercício físico regular, para doentes com claudicação intermitente, } \\
\text { baseado em caminhadas de } 30 \text { a } 60 \text { minutos (três dias por semana no mínimo), devendo o doente } \\
\text { parar e descansar em caso de dor; } \\
\text { - Referenciar para a cirurgia vascular se: IPTB inferior a } 0,8 \text {; sinais e sintomas de infecção; dor } \\
\text { mantida em repouso, mesmo com o membro pendente; ausência de ambos os pulsos pedioso e } \\
\text { tíbial posterior; } \\
\text { - Se IPTB inferior a } 0,5 \text { referenciar com urgência para ser observado pela cirurgia vascular. }\end{array}$ \\
\hline
\end{tabular}

\section{CONCLUSÕES E IMPLICAÇÕES PARA A PRÁTICA DE ENFERMAGEM}

$\mathrm{O}$ alicerce dos cuidados de enfermagem aos clientes com úlcera de perna independentemente da etiologia, tem como base o estabelecimento de uma relação terapêutica que permita a colheita de informação detalhada sobre o cliente e núcleo envolvente, dos problemas que percepciona como seus e o nível de afecção das suas actividades de vida diárias. Para posterior elaboração de um plano de cuidados individualizado, que forneça resposta às suas reais necessidades, ou seja, todo o processo de avaliação e tratamento implica uma abordagem à pessoa, enquanto ser complexo e integral, e não apenas à sua ferida.

Para uma intervenção de enfermagem estruturada e efectivamente centrada na pessoa é, em primeira instância, fundamental mobilizar competências no âmbito da comunicação eficaz e relação interpessoal, que criam um ambiente caloroso propício à individualização de cuidados. Esta personalização ao permitir um profundo conhecimento sobre os padrões de vida habituais, situação social, económica e familiar, as percepções e expectativas sobre o seu estado actual e as preferências está associada a um incremento dos resultados positivos. A promoção de mecanismos de adaptação revelou-se essencial para lidar com a situação de crise, induzida pela doença, com particular destaque para a auto-eficácia e motivação, que permitiu enfrentar a nova situação mais como um desafio, do que como uma ameaça. A existência de apoio social foi o aspecto mais referido pelas pessoas como primordial no seu processo de adaptação, prestado tanto por pessoas significativas, como pelo contacto com pessoas em situação similar (grupos de auto-ajuda) ou pelo enfermeiro. A educação para a auto-gestão da saúde foi considerada de extrema importância na redução da co-morbilidade, ao diminuir os factores de riscos existentes e criar condições fisiológicas favoráveis a uma melhor cicatrização. A monitorização e controlo da dor, a continuidade de cuidados, bem como uma abordagem proporcionada em equipe multiprofissional aumentou a satisfação com o plano se saúde, optimizou a taxa de adesão ao regime terapêutico e fomentou a percepção sobre a qualidade de vida. A formação contínua e actualizada dos enfermeiros prestadores de cuidados à pessoa com úlcera de perna emergiu como outro aspecto positivamente associado à eficácia e excelência das intervenções implementadas. A instituição de tratamento adequado e atempado é uma 
componente indissociável das acções de enfermagem supra-mencionadas, responsável pelo incremento dos ganhos em saúde, que se explicita.

Encontram-se, portanto, expressas linhas de orientação e recomendações para a acção do enfermeiro, com a

\section{REFERÊNCIAS}

1. Ordem dos Enfermeiros. Padrões de qualidade dos cuidados de enfermagem. Lisboa; 2001.

2. Van Hecke A, Grypdonck M, Defloor T. A review of why patients with leg ulcers do not adhere to treatment. J Clin Nurs. 2009;18(3):337-49.

3. Portugal. Instituto Nacional de Estatística. Projecções de população residente em Portugal: 2008- 2060. Lisboa; 2008.

4. Brown A. Does social support impact on venous ulcer healing or recurrence? Br J Community Nurs. 2008;13(3):S6, S8, S10.

5. Werchek S. Diagnosis and treatment of venous leg ulcers. Nurse Pract. 2010;35(12):46-53.

6. Vowden P. Leg ulcers: assessment and management. Indep Nurse. 2010;(1)30-3.

7. Hecke A, Grypdonck M, Defloor T. Interventions to enhance patient compliance with leg ulcer treatment: a review of the literature. J Clin Nurs. 2008;171(1):29-39.

8. Ebbeskog B, Emami A. Older patients' experience of dressing changes on venous leg ulcers: more than just a docile patient. J Clin Nurs. 2005;14(10):1223-31.

9. European Commission. EUROSTAT. Statistics Database. Population projections 2008-2060 [Internet]. [cited 2009 Nov 25]. Available from: http://ec.europa.eu/eurostat

10. Edwards H, Courtney M, Finlayson K, Lindsay E, Lewis C, Chang $A$, et al. Chronic venous leg ulcers: effect of a community nursing intervention on pain and healing. Nurs Stand. 2005;19(52):47-54.

11. Melnyk B, Fineout-Overholt E. Evidence-based practice in nursing \& healthcare: a guide to best practice. Philadelphia: Lippincott Williams \& Wilkins; 2005.

12. Van Hecke A, Grypdonck M, Defloor T. The clinical nursing competences and their complexity in Belgian general hospitals. J Adv Nurs. 2006;56(6):669-78.

13. Forssgren A, Fransson I, Nelzén O. Leg ulcer point prevalence can be decreased by broad-scale intervention: a follow-up cross-sectional study of a defined geographical population. Acta Derm Venereol. 2008;88(3):252-6.

14. Domeij D, Flodén M. Population aging and international capital flows. Intern Econ Rev. 2006;47(3):1013-32. finalidade de transformar este artigo num instrumento de apoio, em contexto do cuidar à úlcera de perna. Embora, se aconselhe a sua adaptação à realidade profissional de cada um, de modo a que a avaliação e intervenção realizada seja a mais apropriada, tendo sempre como foco o cliente, a pessoa.
15. Heinen M, Evers A, Van Uden C, CJM, PCM, Van Achterberg T. Sedentary patients with venous or mixed leg ulcers: determinants of physical activity. J Adv Nurs. 2007;60(1):50-7.

16. Registered Nurses Association of Ontario. Nursing Best Practice Guideline. Assessment and management of venous leg ulcers: complete summary [Internet]. Toronto, Ontario; 2008 [cited 2011 Jan 4]. Available from: http://www.rnao. org/Storage/46/4017_RNAO_Venous_Leg.FINAL.pdf

17. US Department of Health \& Human Services. National Guideline Clearinghouse. Guideline for management of wounds in patients with lower-extremity arterial disease: complete summary [Internet]. Rockville; 2008 [cited 2011 Jan 4]. Available from: http://www.guideline.gov/content. aspx?id=12613

18. US Department of Health \& Human Services. National Guideline Clearinghouse. Guideline for management of wounds in patients with lower-extremity venous disease: complete summary [Internet]. Rockville; 2005. [cited 2011 Jan 4]. Available from: http://www.guideline.gov/content. aspx?id=12613

19. US Department of Health \& Human Services. National Guideline Clearinghouse. Guideline leg ulcer guidelines: a pocket guide for practice: complete summary [Internet]. Rockville; 2007 [cited 2011 Jan 4]. Available from: http://www. guideline.gov/content.aspx?id=9830

20. US Department of Health \& Human Services. National Guideline Clearinghouse. Guideline Summary algorithm for venous ulcer care with annotations of available evidence: complete summary [Internet]. Rockville; 2005. [cited 2011 Jan 4]. Available from: http://www.guideline.gov

21. Zink M, Rousseau P, Holloway G. Lower extremity ulcers: acute and chronic wounds: nursing management. St. Louis: Mosby; 1992. p. 164-212.

22. Hjerppe A, Saarinen J, Venermo M, Huhtala H, Vaalasti A. Prolonged healing of venous leg ulcers: the role of venous reflux, ulcer characteristics and mobility. J Wound Care. 2010;19(11):474-84.

23. Azoubel R, Torres GV, Silva LWS, Gomes FV, Reis LA. Effects of the decongestive physiotherapy in the healing of venous ulcers. Rev Esc Enferm USP [Internet]. 2010 [cited 2011 Jan 4];44(4):1085-92. 